\title{
Carcinoma of Unknown Primary Site Treated with Carboplatin + Paclitaxel + Bevacizumab + Erlotinib and Its Maintenance Chemotherapy
}

\author{
Hirotoshi Yasui ${ }^{a}$ Kazuhide Sato ${ }^{a}$ Yoshihiro Takeyama ${ }^{a}$ Toshio Kato ${ }^{a}$ \\ Hiroyuki Hashimoto $^{a}$ Yasutaka Fukui $^{a}$ Nagashima Yoshihisa $^{b}$ \\ Matsuyoshi Maedac Hideo Gonda ${ }^{a}$ Ryujiro Suzuki ${ }^{a}$ \\ Departments of ${ }^{\mathrm{a}}$ Respiratory Medicine, ${ }^{\mathrm{b}}$ Otorhinolaryngology and ${ }^{\mathrm{C}} \mathrm{Clinical}$ Pathology, \\ Toyohashi Municipal Hospital, Toyohashi, Japan
}

\section{Key Words}

Carcinoma of unknown primary site $\cdot$ Bronchoscopy $\cdot$ Immunohistochemistry $\cdot$ Carboplatin + paclitaxel + bevacizumab + erlotinib $\cdot$ Maintenance chemotherapy

\begin{abstract}
About 3\% of all cancer patients suffer from carcinoma of unknown primary site (CUP). In spite of its rarity, we will encounter them. While CUPs manifest a wide variety of clinical presentations, they have often resulted in poor prognosis. Although platinum/taxane combination chemotherapy, e.g. carboplatin (CBDCA) + paclitaxel (PTX) is widely used for patients suffering from CUP, the response rate is only about $30-40 \%$ and the median overall survival (OS) is only 9 months, which means that improvement is needed. Among the new regimens, the combination of CBDCA, PTX, bevacizumab (BEV) and erlotinib is thought to be highly promising. Herein, we report a case with CUP treated with this regimen and his maintenance therapy. Our patient was a 75-year-old man who was admitted with a left neck lump. CT revealed systemic massive lymphadenopathy. In spite of various investigations for primary origin, he was diagnosed with CUP and treated with CBDCA + PTX + BEV + erlotinib (AUC $6+175 \mathrm{mg} / \mathrm{m}^{2}+15 \mathrm{mg} / \mathrm{kg}+150 \mathrm{mg}$ ). Since the evaluation of the efficacy indicated partial response, maintenance chemotherapy (BEV and erlotinib) was performed. Chemotherapy was continued for 9 months until the patient was in a progressive disease state with meningeal dissemination. He died 12 months after the initiation of chemotherapy, which is a longer period than the previously reported OS. Of note, according to our case, CBDCA + PTX $+\mathrm{BEV}+$ erlotinib and its maintenance chemotherapy are feasible and well tolerated for CUP.
\end{abstract}

(c) 2014 S. Karger AG, Basel 
Yasui et al.: Carcinoma of Unknown Primary Site Treated with Carboplatin + Paclitaxel

+ Bevacizumab + Erlotinib and Its Maintenance Chemotherapy

\section{Introduction}

Carcinoma of unknown primary (CUP) currently accounts for approximately $3 \%$ of all cancer diagnoses. Although empiric chemotherapy with taxane/platinum regimens, e.g. carboplatin (CBDCA) + paclitaxel (PTX) is widely used for CUP patients, no clear evidence exists on the superiority to any other administered regimens. Taxane/platinum regimens yield response rates of 30-40\%; the median overall survival (OS) and the median progression-free survival are 9.0 and 6.0 months with 1 - and 2-year survival rates of approximately 40 and $20 \%$, respectively [1].

During the last several years, new drugs targeting either the angiogenic pathway or the cancer cell proliferation pathway have been used for a variety of cancers, including lung, colon, breast, and pancreatic cancer. Because the lung, colon, and pancreas are often identified as the primary sites by autopsy in CUP patients, it seems that these new drugs are also pertinent in the empiric treatment for such patients. A phase II trial of PTX/CBDCA + bevacizumab (BEV)/erotinib regimen for CUP patients was reported in 2009 [1]. This regimen produced a response rate of $53 \%$, the median OS was 12.6 months, the median progression-free survival was 8 months, and the 1- and 2-year survival rates were 51 and $27 \%$, respectively $[1,2]$. Both the median progression-free survival and a 2-year survival rate are the best of the previously reported empiric chemotherapy regimens. Thus, this regimen seems to be promising for CUP patients. We herein report the case of a CUP patient treated with CBDCA, PTX, BEV and erlotinib and his maintenance therapy.

\section{Case Presentation}

A 60-year-old man presented to his primary care physician with cervical lymphadenopathy persisting for 1 month and was referred to an otolaryngologist at our hospital. The patient did not complain about any other symptoms (performance status: PS 0). He had suffered from a colon polyp, which was treated by endoscopic resection 7 years ago, and had a 40 -year smoking history of 1.5 packs per day.

His whole-body contrast-enhanced CT showed lymphadenopathy of the right cervix, bilateral supraclavicule and mediastinum, emphysema and a small nonspecific node in the right upper lung (fig. 1a, b). His head MRI showed multiple ring and solid enhancing lesions, suggesting brain metastasis (fig. 1c, d). ${ }^{18}$ F-FDG PET/CT demonstrated FDG accumulation in the lymph nodes and a pulmonary node (fig. 1e-g). Serum levels of carcinoembryonic antigen (CEA) were elevated at $16.8 \mathrm{ng} / \mathrm{ml}$ (normal values $<5 \mathrm{ng} / \mathrm{ml}$ ), and no other abnormalities were found (table 1) when investigating for tumor markers.

In order to find a clue for the primary lesion pathologically, needle biopsy of the cervical lymph node was performed. Cytological examination revealed poorly differentiated adenocarcinoma, and immunohistochemistry (IHC) showed the following: cytokeratin (CK) $7(+)$, CK20 (+), MUC1 (+), MUC2 (-), SP-A (-), TTF-1 (-), CD5 (-), CDX2 (-), human gastric mucin (-), ALK mutation (-), and EGFR mutation (-) (fig. 2). Bronchoscopy was performed but cytological examination was negative for bronchial lavage fluid, and upper and lower gastroscopy did not show any abnormality. In spite of these diagnostic approaches for detecting the primary site, it remained unclear, and the patient was diagnosed with CUP.

After approval by the Intramural Ethics Committee and written informed consent had been given, the patient was treated with CBDCA + PTX + BEV + erlotinib (AUC 6 + 175 $\mathrm{mg} / \mathrm{m}^{2}+15 \mathrm{mg} / \mathrm{kg}+150 \mathrm{mg}$ ). After the chemotherapy had started, the serum level of CEA decreased (fig. 3). Since his whole-body CT revealed a decrease in lymph node size after five 
cycles of the regimen, the patient achieved partial response (fig. 1h-k). With this result, he was treated with maintenance chemotherapy of BEV + erlotinib $(15 \mathrm{mg} / \mathrm{kg}+150 \mathrm{mg})$. No clear side effect was detected in blood chemistry after five cycles (table 2). After six cycles of maintenance chemotherapy (i.e. 8 months after the initial chemotherapy), the patient complained of left facial paralysis. We diagnosed that meningeal dissemination caused this facial paralysis, based on the result of a CT/MRI scan which showed a new contrastenhanced area in the left internal auditory canal (fig. 1l-o). This result was suggestive of progressive disease, and thus chemotherapy was stopped. Treatment-related toxicity was grade 3 peripheral neuropathy and grade 1 alanine aminotransferase (ALT) elevation as well as stomatitis, skin rash, and dysgeusia; he did not have any hematologic toxicity. Peripheral neuropathy was considered to be possibly related to PTX and indeed improved after CBCDA and PTX treatment was finished.

Further chemotherapy could not be performed due to the patient's worsening performance states (PS 3). In order to palliate his facial paralysis, neck pain and facial edema due to jugular venous distention by lymphadenopathy, cranial irradiation and radiation therapy for neck lymphadenopathy were performed. Our patient died of CUP 12 months after the initial chemotherapy (fig. 3).

\section{Discussion}

A primary tumor may not be detected because of its extremely small size or possible local regression due to antitumor immune defenses as well as its protracted clinical latency [3]. Since it is difficult to detect the organs where the primary tumor is located, investigation with imaging, tumor marker and IHC markers is performed to at least classify the type of carcinomas. CUP is classified into four major histopathological subtypes: well- or moderately differentiated adenocarcinomas (50\%); undifferentiated or poorly differentiated adenocarcinomas or carcinomas (30\%); squamous cell carcinomas (15\%), and undifferentiated neoplasms (5\%) [4]. In our case, neither CT, MRI and PET images nor endoscopy detected the primary site. IHC examinations showed adenocarcinoma, and positivity of CK7/CK20 suggested transient cell carcinoma, pancreatic ductal carcinoma, cholangiocellular carcinoma and gastric adenocarcinoma. MUC1 overexpresses in serous-type adenocarcinoma, such as breast or pancreatic cancer, MUC2 tends to overexpress in mucinous adenocarcinoma, such as colon, small intestine and bronchus carcinoma. CK7/CK20 results and MUC1 positivity and MUC2 negativity might indicate that our patient's primary tumor was pancreatic adenocarcinoma. Because other IHCs and examinations were not consistent in determining the primary tumor site, the patient was diagnosed with CUP.

Treatments for CUP patients have so far been empirical. Although data from phase III trials are lacking, regimens with new chemotherapeutic agents (e.g. taxanes, gemcitabine, irinotecan) show a modest improvement; most of these new regimens report response rates of $30-50 \%$ and a median OS of 8-10 months [5, 6]. CBDCA, PTX, BEV and erlotinib regimens attempt to incorporate molecular targeted agents with the existing empirical first treatment for CUP. BEV, a monoclonal antibody targeting vascular epithelial growth factor (VEGF), which inhibits neoangiogenesis, is used alone or in combination with other anticancer drugs in patients with advanced colon, lung, breast, renal, and ovarian cancer [7-10]. Erlotinib, an intracellular EGFR tyrosine kinase inhibitor, prolongs survival in lung and pancreatic cancer $[11,12]$. Preclinical studies suggested that inhibition of EGFR also resulted in the suppression of VEGF levels and might allow the additional inhibition of the angiogenesis pathway. Moreover, blocking of VEGF receptor and EGFR signaling could lead to the primary tumor 
overcoming its acquired resistance to EGFR inhibitors [2, 13]. Clinically, anti-EGFR and antiVEGF combination therapy is well tolerated and effective in several malignancies [2, 13-15]. The combination regimen seemed to be tolerable and more effective than general CUP prognosis. However, the use of these four agents resulted in an expensive treatment. Further study is necessary regarding the cost-benefit of this treatment.

\section{Conclusions}

We reported the case of a CUP patient treated with CBDCA, PTX, BEV and erlotinib and its maintenance chemotherapy. According to our case, CBDCA + PTX + BEV + erlotinib and its maintenance chemotherapy are feasible and well tolerated for CUP.

\section{Acknowledgement}

We are grateful to Dr. Suganuma, Dr. Mashimo and Dr. Mitake for their writing advice. This work was supported by CJLSG (Central Japan Lung Study Group).

\section{Disclosure Statement}

The authors have no conflicts of interest to declare.

\section{References}

1 Huebner G, Link $\mathrm{H}$, Kohne $\mathrm{CH}$, et al: Paclitaxel and carboplatin vs gemcitabine and vinorelbine in patients with adeno- or undifferentiated carcinoma of unknown primary: a randomised prospective phase II trial. $\mathrm{Br}$ J Cancer 2009;100:44-49.

-2 Hainsworth JD, Spiqel DR, Thompson DS, et al: Paclitaxel/carboplatin plus bevacizumab/erlotinib in the first-line treatment of patients with carcinoma of unknown primary site. Oncologist 2009;14:1189-1197.

-3 Alberti C: Carcinoma of unknown primary (CUP); some considerations about pathogenesis and diagnostic strategy, particularly focusing on CUPS pertaining to the Urology. G Chir 2012;33:41-46.

4 Pavilidis N, Pentheroudakis G: Cancer of unknown primary site: 20 questions to be answered. Ann Oncol 2010;21(suppl 7):vii303-vii307.

5 Creco FA, Rodriguez GI, Shaffer DW, et al: Carcinoma of unknown primary site: sequential treatment with paclitaxel/carboplatin/etoposide and gemcitabine/irinotecan: a Minnie Pearl Cancer Research Network phase II trial. Oncologist 2004;9:644-652.

-6 Pouessel D, Culine S, Becht C, et al: Gemcitabine and docetaxel as frontline chemotherapy in patients with carcinoma of unknown primary site. Cancer 2004;100:1257-1261.

7 Yang JC, Haworth L, Sherry RM, et al: A randomized trial of bevacizumab, an anti-vascular endothelial growth factor antibody, for metastatic renal cancer. N Engl J Med 2003;349:427-434.

-8 Hurwitz H, Fehrenbacher L, Novotny W, et al: Bevacizumab plus irinotecan, fluorouracil, and leucovorin for metastatic colorectal cancer. N Engl J Med 2004;350:2335-2342.

-9 Sandler AB, Gray R, Perry MC, et al: Paclitaxel-carboplatin alone or with bevacizumab for non-small-cell lung cancer. N Engl J Med 2006;355:2542-2550.

$\longrightarrow 10$ Miller KD, Wang M, Gralow J, et al: Paclitaxel plus bevacizumab versus paclitaxel alone for metastatic breast cancer. N Engl J Med 2007;357:2666-2676.

$\checkmark 11$ Moore MJ, Goldstein D, Hamm J, et al: Erlotinib plus gemcitabine compared with gemcitabine alone in patients with advanced pancreatic cancer: a phase III trial of the National Cancer Institute of Canada Clinical Trials Group. J Clin Oncol 2007;25:1960-1966.

12 Rosell R, Carcereny E, Gervalis R, et al: Erlotinib versus standard chemotherapy as first-line treatment for European patients with advanced EGFR mutation-positive non-small-cell lung cancer (EURTAC): a multicenter, open-label, randomized phase 3 trial. Lancet Oncol 2012;13:239-246. 


\section{Case Reports in Oncology}

\begin{tabular}{l|l}
\hline Case Rep Oncol 2014;7:583-590 \\
\hline DOI: $10.1159 / 000366268$ & $\begin{array}{l}\text { C 2014 S. Karger AG, Basel } \\
\text { www.karger.com/cro }\end{array}$ \\
\hline
\end{tabular}

Yasui et al.: Carcinoma of Unknown Primary Site Treated with Carboplatin + Paclitaxel

+ Bevacizumab + Erlotinib and Its Maintenance Chemotherapy

13 Herbst RS, Ansari R, Bustin F, et al: Efficacy of bevacizumab plus erlotinib versus erlotinib alone in advanced non-small-cell lung cancer after failure of standard first-line chemotherapy (BeTa): a double-blind, placebocontrolled, phase 3 trial. Lancet 2011;377:1846-1854.

14 Tournigand C, Samson B, Scheithauer W, et al: Bevacizumab (Bev) with or without erlotinib as maintenance therapy, following induction first-line chemotherapy plus Bev, in patients (pts) with metastatic colorectal cancer (mCRC): efficacy and safety results of International GERCOR GREAM Phase III trial (abstract). J Clin Oncol 2012;30(suppl 18):LBA3500.

15 Van Cutsem E, Vervenne WL, Bennouna J, et al: Phase III trial of bevacizumab in combination with gemcitabine and erlotinib in patients with metastatic pancreatic cancer. J Clin Oncol 2009;27:2231-2237.

Table 1. Blood biochemistry and tumor markers before the therapy; CEA was elevated, and no other abnormalities were found

\begin{tabular}{llllll}
\hline WBC & $6,900 / \mu \mathrm{l}$ & $\mathrm{TP}$ & $7.0 \mathrm{~g} / \mathrm{dl}$ & $\mathrm{CEA}$ & $16.8 \mathrm{ng} / \mathrm{ml}$ \\
Neutrophils & $60.6 \%$ & AST & $25 \mathrm{U} / \mathrm{l}$ & $\mathrm{SCC}$ & $0.9 \mathrm{ng} / \mathrm{ml}$ \\
Lymphocytes & $30.0 \%$ & ALT & $36 \mathrm{U} / \mathrm{l}$ & $\mathrm{SLX}$ & $36.1 \mathrm{U} / \mathrm{ml}$ \\
Monocytes & $7.0 \%$ & $\mathrm{LDH}$ & $267 \mathrm{U} / \mathrm{l}$ & $\mathrm{NSE}$ & $9.8 \mathrm{ng} / \mathrm{ml}$ \\
Eosinophils & $2.0 \%$ & ALP & $233 \mathrm{U} / \mathrm{l}$ & $\mathrm{AFP}$ & $3.2 \mathrm{ng} / \mathrm{ml}$ \\
Basophils & $0.4 \%$ & $\gamma \mathrm{GTP}$ & $32 \mathrm{U} / \mathrm{l}$ & $\mathrm{CA} 19-9$ & $12.9 \mathrm{U} / \mathrm{ml}$ \\
& & T-Bil & $0.6 \mathrm{mg} / \mathrm{dl}$ & $\mathrm{PSA}$ & $0.853 \mathrm{ng} / \mathrm{ml}$ \\
$\mathrm{RBC}$ & $486 \times 10^{4} / \mu \mathrm{l}$ & $\mathrm{BUN}$ & $20 \mathrm{mg} / \mathrm{dl}$ & $\mathrm{sIL} 2-\mathrm{R}$ & $443 \mathrm{U} / \mathrm{ml}$ \\
$\mathrm{Hb}$ & $15.2 \mathrm{~g} / \mathrm{dl}$ & $\mathrm{Cre}$ & $0.82 \mathrm{mg} / \mathrm{dl}$ & & \\
$\mathrm{Ht}$ & $45.3 \%$ & $\mathrm{Na}$ & $142 \mathrm{mEq} / \mathrm{l}$ & & \\
$\mathrm{Plt}$ & $23.2 \times 10^{4} / \mu \mathrm{l}$ & $\mathrm{K}$ & $4.6 \mathrm{mEq} / \mathrm{l}$ & & \\
& & $\mathrm{Cl}$ & $105 \mathrm{mEq} / \mathrm{l}$ & & \\
& & $\mathrm{CRP}$ & $0.22 \mathrm{mg} / \mathrm{dl}$ & & \\
\hline
\end{tabular}

$\mathrm{TP}=$ total protein; $\mathrm{ALP}=$ alkaline phosphatase $\gamma \mathrm{GTP}=\gamma$-glutamyl transpeptidase $; \mathrm{T}$-Bil $=$ total bilirubin; SCC = squamous cell carcinoma antigen; SLX = sialyl lewis X-i antigen ; NSE = neuron-specific $\gamma$-enolase; $\mathrm{AFP}=\alpha$-fetoprotein.

Table 2. Blood biochemistry after five cycles of CBDCA + PTX + BEV + erlotinib; no side effects and abnormalities were detected

\begin{tabular}{llll}
\hline WBC & $7,890 / \mu \mathrm{l}$ & TP & $6.3 \mathrm{~g} / \mathrm{dl}$ \\
Neutrophils & $73.0 \%$ & AST & $23 \mathrm{U} / \mathrm{l}$ \\
Lymphocytes & $21.0 \%$ & ALT & $38 \mathrm{U} / \mathrm{l}$ \\
& & T-Bil & $0.8 \mathrm{mg} / \mathrm{dl}$ \\
$\mathrm{RBC}$ & $288 \times 10^{4} / \mu \mathrm{l}$ & $\mathrm{BUN}$ & $24 \mathrm{mg} / \mathrm{dl}$ \\
$\mathrm{Hb}$ & $10.7 \mathrm{~g} / \mathrm{dl}$ & $\mathrm{Cre}$ & $0.99 \mathrm{mg} / \mathrm{dl}$ \\
$\mathrm{Ht}$ & $34.1 \%$ & $\mathrm{Na}$ & $141 \mathrm{mEq} / \mathrm{l}$ \\
$\mathrm{Plt}$ & $19.1 \times 10^{4} / \mu \mathrm{l}$ & $\mathrm{K}$ & $4.5 \mathrm{mEq} / \mathrm{l}$ \\
& & $\mathrm{Cl}$ & $103 \mathrm{mEq} / \mathrm{l}$ \\
& & $\mathrm{CRP}$ & $0.03 \mathrm{mg} / \mathrm{dl}$ \\
\hline
\end{tabular}

For the abbreviations used, refer to table 1. 


\section{Case Reports in Oncology}

\begin{tabular}{l|l}
\hline \multicolumn{2}{l}{ Case Rep Oncol 2014;7:583-590 } \\
\hline DOI: $10.1159 / 000366268$ & $\begin{array}{l}\text { C 2014 S. Karger AG, Basel } \\
\text { www.karger.com/cro }\end{array}$ \\
\hline
\end{tabular}

Yasui et al.: Carcinoma of Unknown Primary Site Treated with Carboplatin + Paclitaxel

+ Bevacizumab + Erlotinib and Its Maintenance Chemotherapy

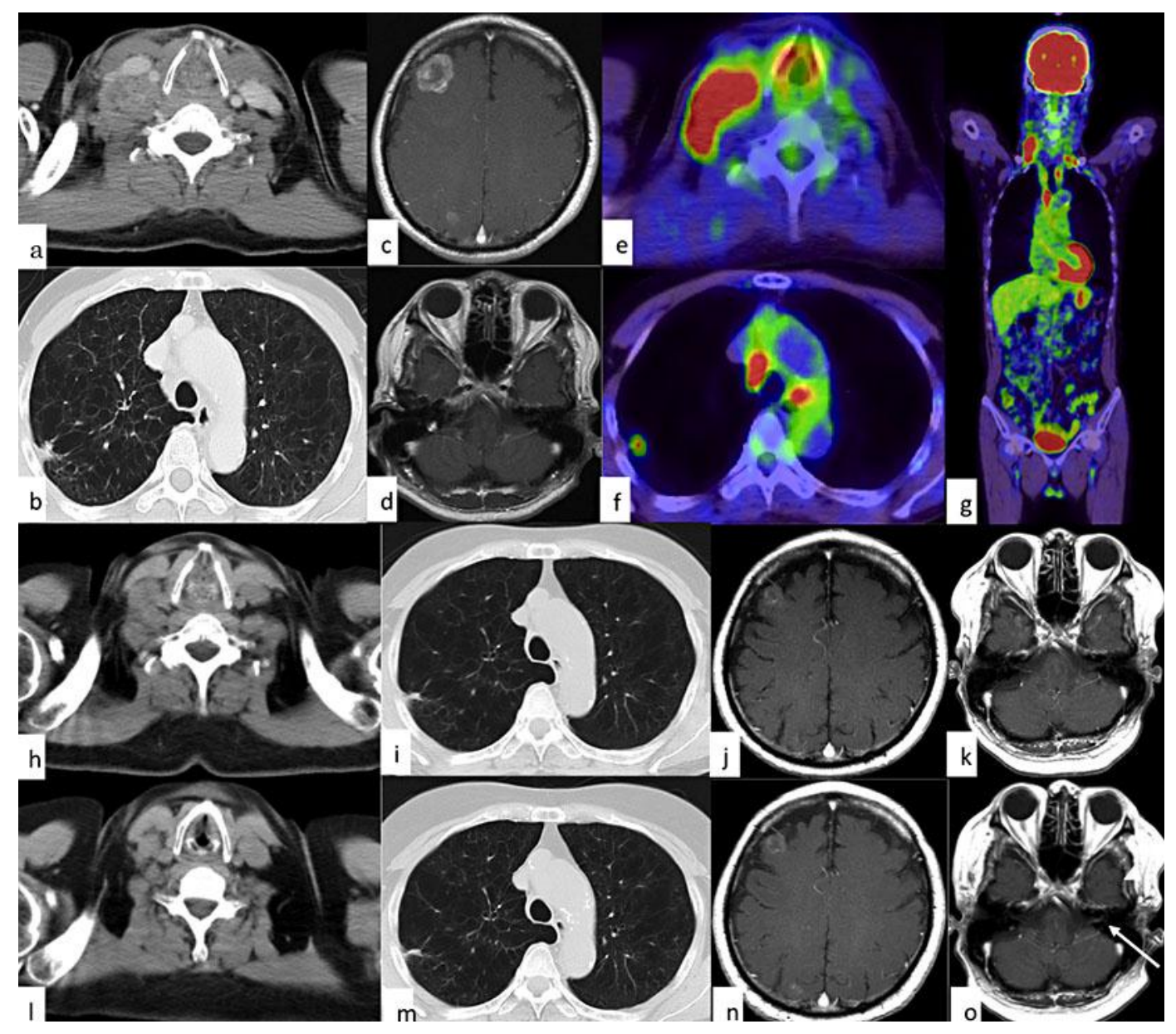

Fig. 1. a, b Lymphadenopathy of the right cervix and a small nonspecific node in the right upper lung on CT. c, d Metastasis in the right frontal and parietal lobe and no lesion in the internal auditory canals on head MRI. e-g Multiple metastatic lesions in the cervical lymph nodes, a pulmonary node, and accumulation in cervical and mediastinal lymph nodes on PET-CT. No other accumulation was observed except a pulmonary node. $\mathbf{h}-\mathbf{k}$ Response on CT after five cycles of CBDCA, PTX, BEV, and erlotinib. Cervical lymph nodes decreased in size (h), but the pulmonary node did not change (i). Head metastasis was reduced (j) and no new lesion was observed (k). I-o Evaluation after six cycles of maintenance with BEV and erlotinib. CT and MRI (T1 weighted with gadolinium) showed no specific changes in each of the lesions except the appearance of a contrast-enhanced area in the left internal auditory canal (arrow). 


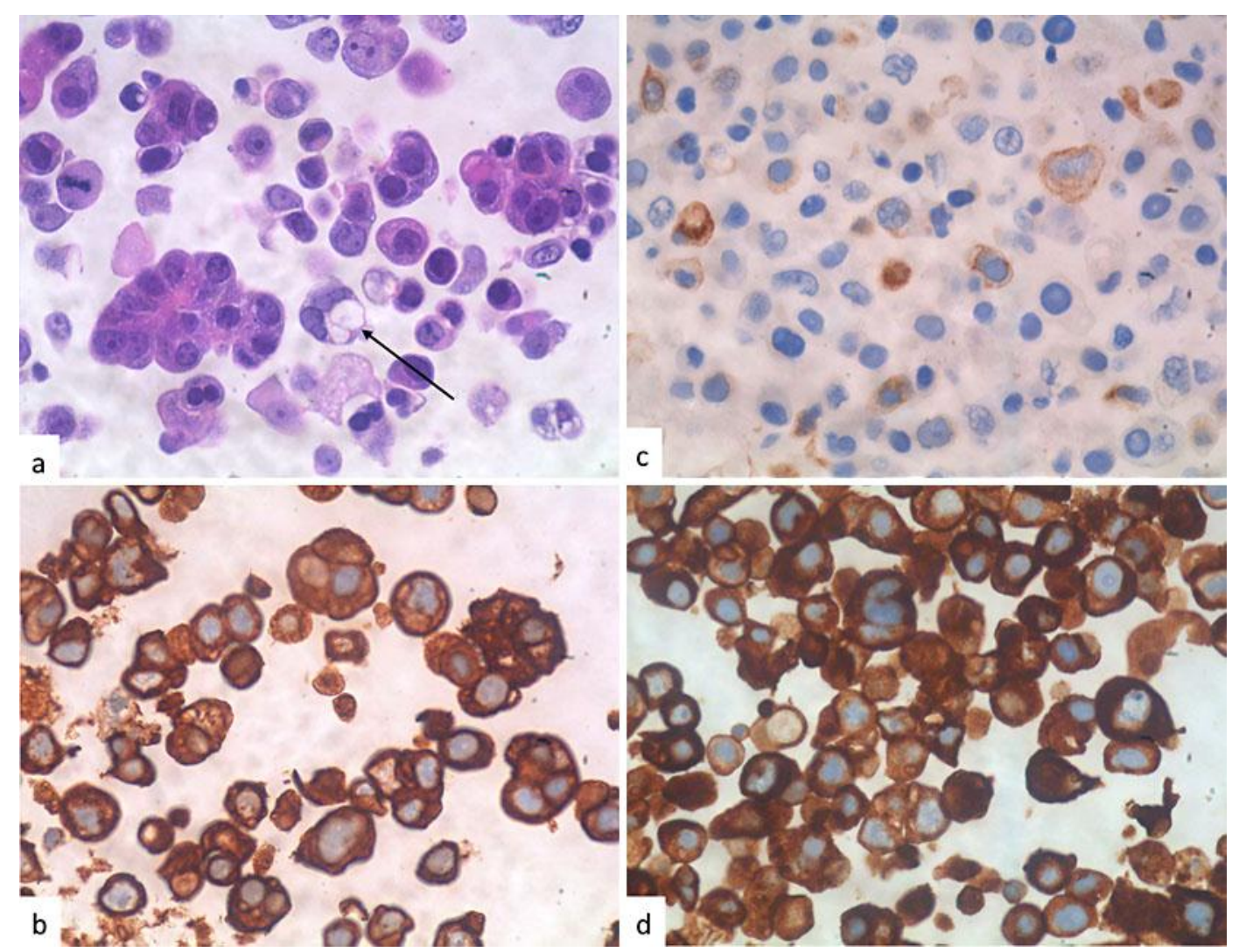

Fig. 2. Cytological examination of cervical lymph node needle biopsy. Original magnifications. $\times 400$. a HE stain; the arrow indicates the duct of the gland. IHC staining was positive for CK7 (b), CK20 (c), and MUC1 (d). 


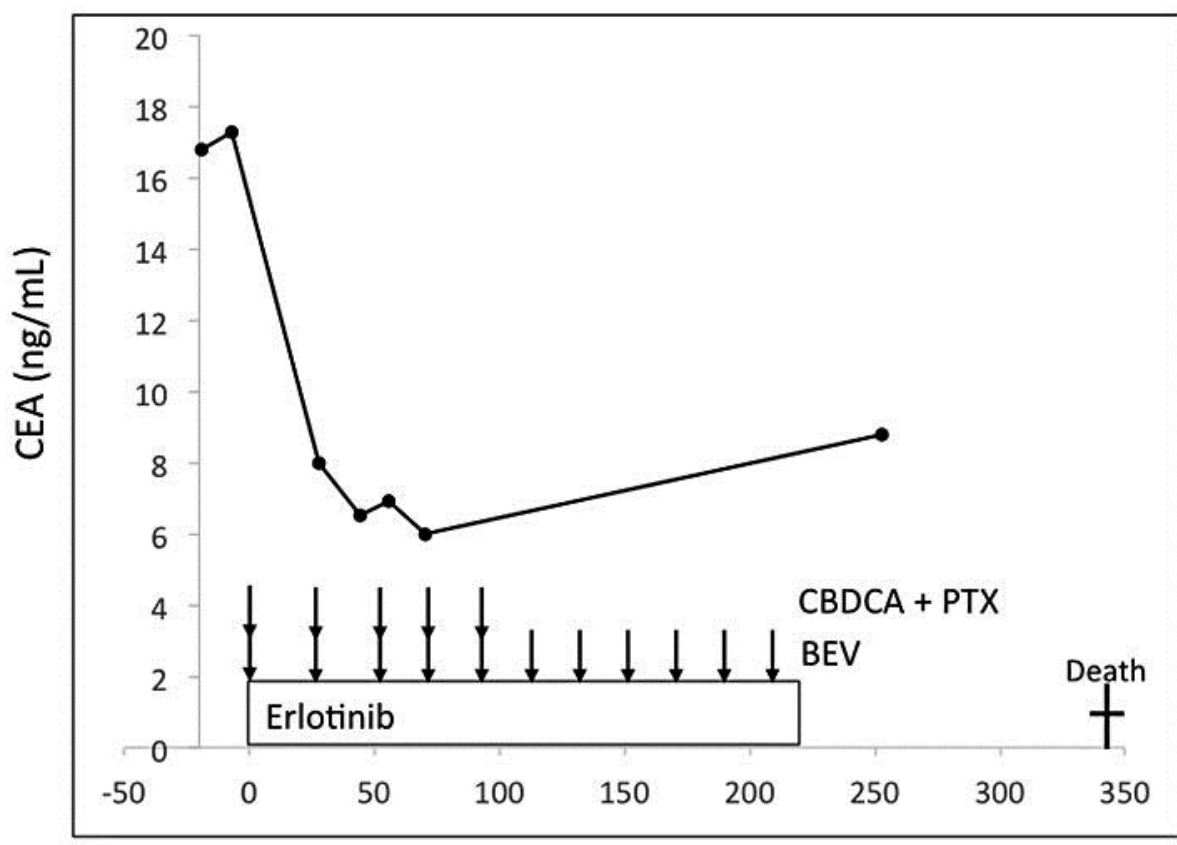

Days after chemotherapy

Fig. 3. Overview of the therapy and change of CEA. The arrows show chemotherapies (CBDCA, PTX, and $\mathrm{BEV}$ ) and the bar shows erlotinib. 